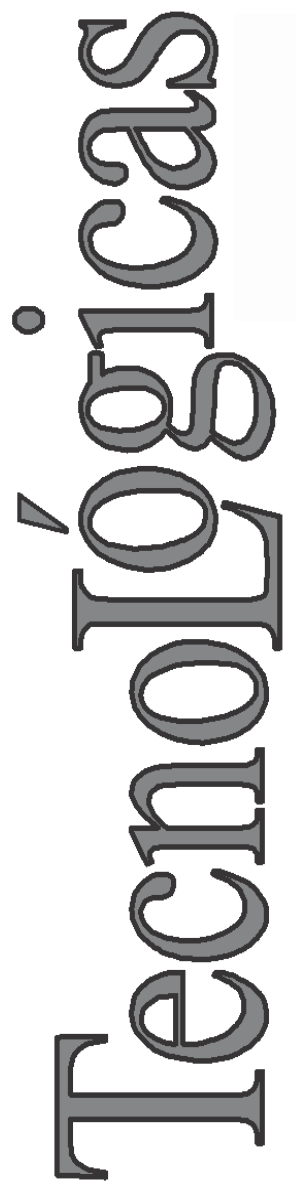

\title{
Análisis de Redes MPLS/BGP/VPN en el Rendimiento de los Anuncios del Algoritmo de Enrutamiento BGP
}

\author{
Analysis of MPLS/BGP/VPN \\ Performance Advertising BGP \\ Routing Algorithm
}

\author{
Mónica Espinosa-Buitrago ${ }^{1}$ \\ Octavio Salcedo-Parra ${ }^{2}$ \\ Ricardo Gómez-Vargas ${ }^{3}$
}

1 Ingeniería de Telecomunicaciones, Universidad Santo Tomas, Bogotá-Colombia monica.espinosa@usantotomas.edu.co 2 Ingeniería Electrónica,

Universidad Distrital, Bogotá-Colombia osalcedo@udistrital.edu.co 3 Ingeniería de Telecomunicaciones, Universidad Santo Tomas, Bogotá-Colombia ricardogomez@usantotomas.edu.co correo 


\section{Resumen}

Los routers de borde del proveedor de servicios establecen VPNs (Virtual Private Network) realizando el enrutamiento entre los usuarios y la red de núcleo (Core) por medio de Routers Virtuales (VR). Los routers virtuales establecen el enrutamiento a las redes MPLS/VPN/BGP por medio de las tablas VRF (Virtual Router Forwarding), las cuales utilizan el algoritmo de enrutamiento Borde Gateway Protocol (BGP) para sus anuncios. Las sesiones BGP son del tipo malla completa en donde se establecen mayores costos en los anuncios, por ello se realiza un análisis comparativo con rutas reflejadas, obteniendo un $31 \%$ de mejora a nivel de los costos del algoritmo, en la configuración de rutas reflejadas en la función de encapsulamiento MPLS (Multiprotocol Label Switching).

\section{Palabras clave}

MPLS; VPN; BGP; anuncios; rendimiento.

\section{Abstract}

The provider edge router of the service provider establishes VPNs (Virtual Private Network) performing routing between users and core network through the virtual routers (VR). The virtual routers develop routing to the networking MPLS/VPN/BGP through VRF (Virtual Router Forwarding), which use the routing algorithm Edge Gateway Protocol (BGP) for your advertising, therefore performed a comparative analysis with routes reflector, obtaining a $31 \%$ improvement at the cost of the algorithm, in shaping the role routes reflector in MPLS encapsulation.

\section{Keywords}

MPLS; VPN; BGP; advertising; performance. 


\section{INTRODUCCIÓN}

Los proveedores de servicios SP (Service Provider) realizan conexiones a nivel del usuario final por medio de las redes virtuales privadas (VPN), esta técnica a obtenido un rápida evolución en los últimos 10 Años en el estudio de (Ming, 2012), al igual que los ambientes como enrutamiento IP, por ello los modelos de VPNs igual a igual como se muestra en el estudio de (Palmiery, 2003) han sido adoptados por múltiples compañías, que permiten distinguir y separar el tráfico por medio de etiquetas MPLS (Multiprotocol Label Swithing), teniendo una excelente planificación y expansión con diferentes aplicaciones extendidas de red en (Easo, 1955) ; gracias al incremento porcentual de usuarios y las nuevas necesidades entre ellas sistemas de monitoreo en los estudios de (Liwen, 2008) (FengJie, 2011) y sistemas de gobierno en línea en el estudio de (Wei, Fang, Dai, Han, \& Xu, 2006), (Peng, 2009), surge la necesidad de caracterizar las redes MPLS/VPN/BGP, con mecanismos de mejora del rendimiento en este caso las rutas reflejadas. Para ello en este artículo se realiza un análisis de las redes IP-MPLS, de las redes MPLS/BGP/VPN y la implementación de un modelo que permite realizar el análisis comparativo de las técnicas. Obteniendo una la verificación del modelo matemático por medio de un escenario de la red, analizando las funciones de extracción e imposición de etiquetas MPLS en las VRFs, comprobando la mejora del rendimiento de la red a nivel de los costos en los anuncios del algoritmo de enrutamiento BGP.

\section{RED MPLS/BGP/VPN}

Los Elementos de la Red MPLS/VPN/BGP son el Customer Edge (CE), Provider Edge (PE) y Provider Core (P), El Backbone MPLS se compone por los Routers PE y P a nivel del cliente se tiene el router $\mathrm{CE}$. El router $\mathrm{PE}$ es el elemento que tiene contacto directo con las Red del cliente y el router $\mathrm{P}$ es interno a la red MPLS el cual no tienen contacto los clientes directamente. Los routers $\mathrm{PE}$ y $\mathrm{P}$ trabajan en modo de conmutación de etiquetas en los cuales se construyen caminos (LSP) los cuales usan un protocolo 
de distribución de etiquetas (LDP), cuando un PE envía una dirección VPN a través de la red MPLS para identificar el grupo de la VPN la red le asigna un label especifico y asigna también una etiqueta exterior, identificando el PE de salida. La Etiqueta en el interior de la Red es utilizada por el PE de Salida para determinar el puerto de la VPN al que el paquete debe ser direccionado en (Rekhter, 2006), El router CE (Customer Edge) y el router PE (Provider Edge) soporta múltiples niveles de enrutamiento y sus tablas de enrutamiento son llamadas VRF. Las VRFs son lógicamente independientes y pueden llegar a contener traslape de direcciones en otras VRFs. La VPN se forma mediante la definición del cliente que accede a ser miembro de una VRF y este se encuentra en la tabla formada por los sitios que el router PE ha creado. El router PE hace uso de MBGP (Multiprotocol Border Gateway Protocol) para la propagación de la información acerca de las rutas de la VPNs, así como las etiquetas en el interior de MPLS. Para este procedimiento el router $\mathrm{PE}$ debe identificar que el paquete IP que ingresa al nodo pertenezca a la VRF, para el tráfico entrante y trafico saliente del router $\mathrm{PE}$; por lo tanto, el router $\mathrm{PE}$ hace una búsqueda sobre la tabla de rutas IP asociados a la VRF. Si no hay VRF vinculada a una interfaz, el router PE utilizará la ruta predeterminada. Teniendo el tráfico de entrada en el diagrama de flujo de la Fig. 1 (Pico, 2008).

En el diagrama de flujo se especifica que pueden existir tres tipos de resultados de la búsqueda VRF. Si el destino es otro CE, se atribuye al mismo router $\mathrm{PE}$, el $\mathrm{PE}$ remitirá el paquete IP directamente. Si el paquete IP debe recorrer la red del proveedor de servicios, el resultado de este será la búsqueda del atributo 'BGP NextHop', representando el siguiente salto que será establecido en el algoritmo BGP. En el caso de que para el atributo 'BGP NextHop' esté conectado directamente a la entrada PE, este PE el modo directo utiliza sólo una "Encabezado VPN V4" ruta para el tráfico a su destino y sólo se utilizan en los routers PE. Por último, el 'BGP NextHop' puede ser un router PE alcanzado a lo largo de uno o más nodos $\mathrm{P}$, en este caso, el proveedor de la red deberá establecer un túnel entre cada par de PE nodos para el intercambio de paquetes por medio del protocolo LDP (Label Distribucion Protocol) para la conformar caminos LSP (Label-Switched Paths) con 
el fin de evitar el uso de etiquetas VPN en routers P. La entrada PE utiliza una etiqueta vía VPN y un "Encabezado VPN V4".

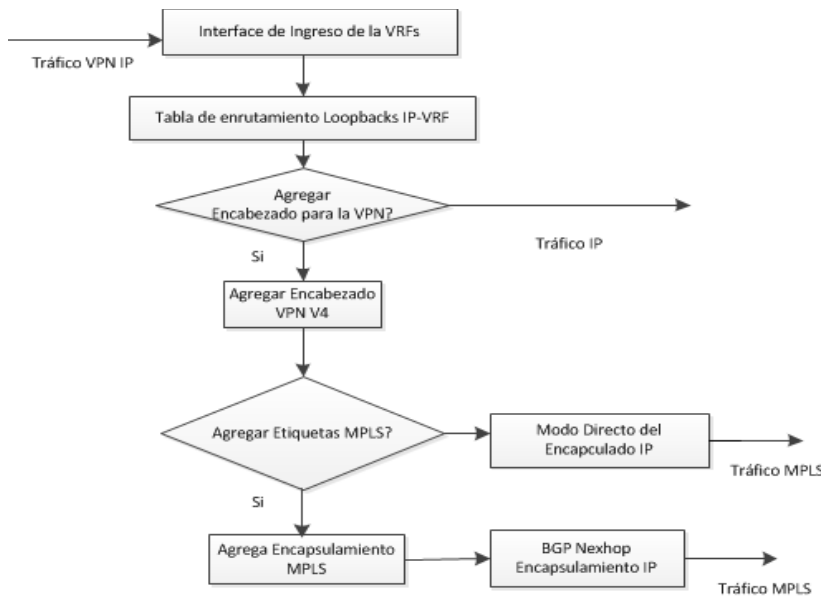

Fig. 1. Función de agregar etiquetas MPLS

En la Fig. 2 (Pico, 2008) se muestra los correspondientes diagramas de flujo que resume el comportamiento del router PE cuando llega el tráfico MPLS, para ello se deben extraer las etiquetas, lo que se considera función POP. Teniendo así, que la información se propaga del PE local a todos los demás Routers PE en la red utilizando BGP. Para garantizar la unicidad de prefijos de diferentes VPNs, un identificador único llama la ruta distinguisher (RD) que esta al comienzo del prefijo, creando una nueva familia de direcciones para VPN IPv4 (Minei I, 2007).

\section{RUTAS REFLEJADAS}

Típicamente, todos las conexiones BGP en un sistema autónomo deben tener una configuración malla completa (full mesh), con ello las información es re-distribuida en todo el sistema autónomo. Para $n$ conexiones BGP en un sistema autónomo se requiere $n *(n-$ 1) / 2 sesiones IBGP en (Feamster, 2005). Esta configuración en los sistemas autónomos tiene serios inconvenientes de escalabilidad en (Feamster, 2005) (Marques, 2005) (Gottlied, 2003). Las rutas 
reflejadas es una técnica trabajada en BGP que busca dar solución a los sistemas malla completa, considerando la aplicación de la Fig. 3, en ASX, hay tres conexiones IBGP con los routers RTR-A, RTR-B y RTR-C. RTA recibe una ruta externa y selecciona la mejor conexión que puede anunciar la ruta sea RTR-B o RTR-C. RTR-B y RTR-C no redistribuirán esta rutas IBGP a otras conexiones IBGP (Bates, 2006). Si se hace esta regla más flexible al router RTR-C le es permitido anunciar las rutas aprendidas de sus conexiones, entonces las rutas IBGP aprendidas de RTR-A al RTR$\mathrm{B}$ y viceversa podrían ser reanunciadas (o reflejadas). Esto podría eliminar la necesidad de establecer una sesión IBGP entre RTR-A y RTR-B en (Bates, 2006) como se muestra en la Fig. 4.

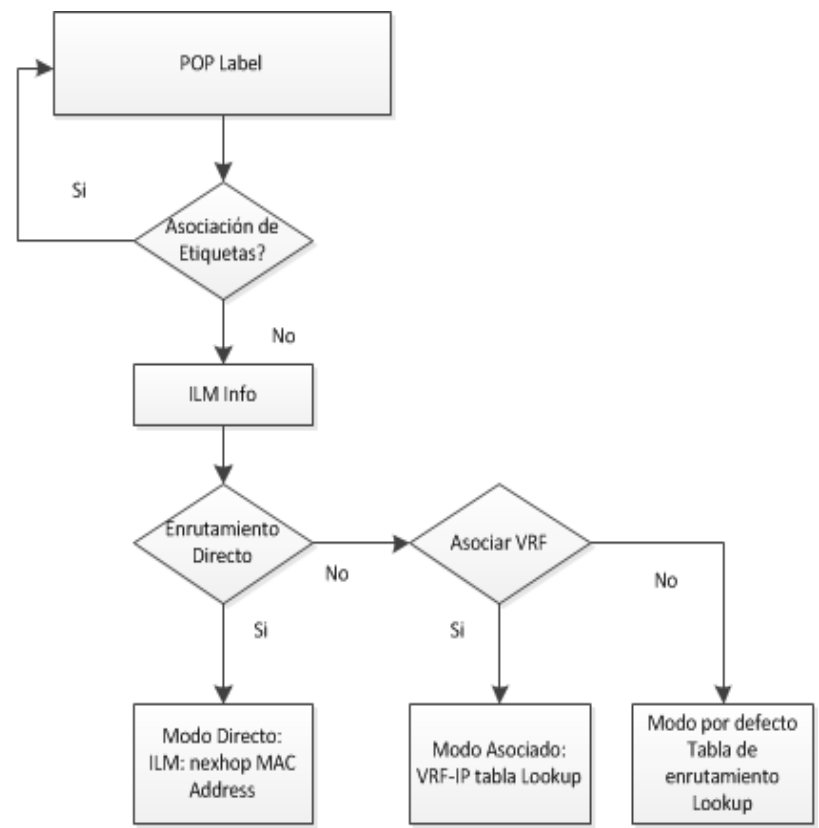

Fig. 2. Función de extracción de etiquetas MPLS 


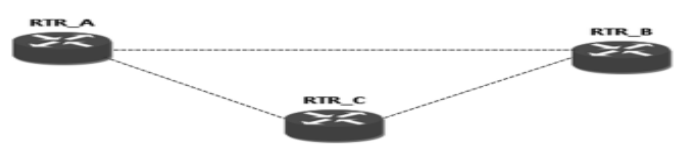

Figura 3 Configuración BGP malla completa

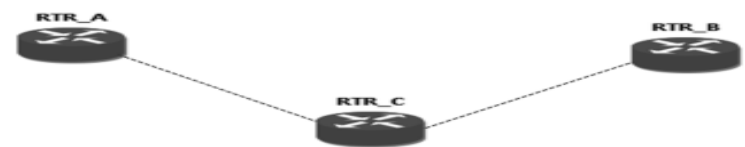

Figura 4 Configuración BGP rutas reflejadas

\section{CARACTERIZACIÓN DE LOS COSTOS DEL ALGORITMO DE ENRUTAMIENTO BGP PARA ROUTERS DE BORDE DE LA RED MPLS/BGP/VPN}

$G=(V, E)$ donde $V=\{1,2, \ldots n\}$ en (Yuri, 2003) denota el número de nodos en la red del sistema autónomo y $E$ son los las líneas de conexión en los routers. Cada línea de conexión tiene un peso asociado, que representa el mensaje de tráfico usado para la malla I-BGP. En la red los nodos $C \underline{C} V$ en (Yuri, 2003) son identificados como clientes del reflector de rutas, estos representan básicamente a las routers de borde que son vecinos dentro de un sistema autónomo. Se denota la longitud como la longitud del camino, entre los nodos $i$ y $j$ en $G$, en una malla completa I-BGP para los clientes $C$ igual a (1) en (Yuri, 2003).

$\sum_{i \in C} \sum_{j \in C} \frac{d(i, j)}{2}$

Definiendo en el sistema autónomo un $R \underline{C}$ Vque ha sido dedicado a ser el router reflector de rutas y es denotado $r(i)$ el servidor de rutas del nodo $i$. Entonces el costo de la sesiones I-BGP es reducido a (2) en el estudio de (Yuri, 2003) este costo es drásticamente menor que en el sistema en el sistema I-BGP malla completa. 
$\sum_{i \in C} d(i, r(i))+\sum_{i \in R} \sum_{j \in R} d(i, j) / 2$

\subsection{Escenario Red MPLS/BGP/VPN}

La función de extracción de etiquetas MPLS para configuración malla completa es validado por medio del snnifer wireshark versión 1.6.5, como se observa en la Fig. 5.

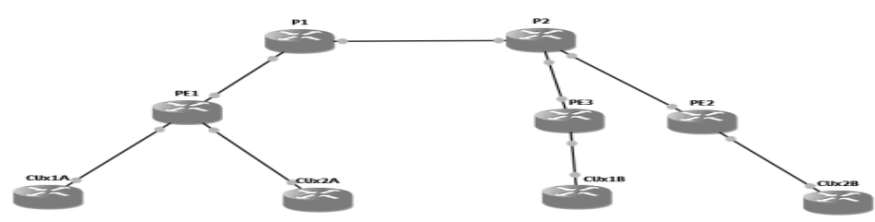

Fig. 5. Escenario MPLS/BGP/VPN

Como se observa en la Fig. 6, se desarrolló un análisis con filtros en wireshark validando las conexiones BGP UPDATE que permite hacer revisión de los anuncios de los routers de borde en la recomendación de $(\mathrm{Li}, 1995)$ que no tienen encapsulamiento MPLS. Como se observa en la Fig. 7, se desarrolló un análisis con filtros en wireshark, validando las conexiones BGP UPDATE para los routers de borde que tienen encapsulamiento MPLS (3).

$\sum_{i \in C} \sum_{j \in C} d(i, j) / 2=955$ Bytes

Como se observa en la Fig. 8, se desarrolló un análisis con filtros en wireshark validando las conexiones BGP UPDATE que no tienen encapsulamiento MPLS aplicando rutas reflejadas para los routers de borde, los mensajes fueron capturados desde el router reflector de rutas el router P1, desarrollado en (4) (5) y (6).

$\sum_{i \in C} \sum_{j \in C} d(i, j) / 2=640$ Bytes 


\begin{tabular}{|c|c|c|c|c|}
\hline \multicolumn{3}{|c|}{ Filter: tcp.port $==53483$ and bgp.type $==2$ and not $\mathrm{mpls}$} & & - Expression... Clear Apply \\
\hline \multirow{3}{*}{ No. } & Time & Source & Destination & Protocol Length Info \\
\hline & 4578.765000 & 172.16 .5 .10 & 172.16 .4 .10 & 160 UPDATE Message \\
\hline & 7393.632000 & $172.16 \cdot 5.10$ & 172.16 .4 .10 & 160 UPDATE Message \\
\hline Filter: & tcp.port $==53483$ ar & d bgp.type $==2$ and not $\mathrm{mpls}$ & & - Expression... Clear Apply \\
\hline \multirow[t]{3}{*}{ No. } & Time & Source & Destination & Protocol Length Info \\
\hline & 15786.237000 & 172.16 .4 .10 & 172.16 .5 .10 & 160 UPDATE Message \\
\hline & 176100.386000 & 172.16 .4 .10 & 172.16 .5 .10 & 160 UPDATE Message \\
\hline Filter: & tcp.port $==32597$ anc & bgp.type $==2$ and not $\mathrm{mpls}$ & & - Expression... Clear Apply \\
\hline & Time & Source & Destination & Protocol Length Info \\
\hline \multicolumn{2}{|c|}{16299.036000} & 172.16 .5 .10 & 172.16 .1 .10 & BGP 160 UPDATE Message \\
\hline \multicolumn{2}{|c|}{191113.872000} & 172.16 .5 .10 & 172.16 .1 .10 & 160 UPDATE Message \\
\hline Filter: & tcp.port $==12174$ an & d bgp.type $==2$ and not $\mathrm{mpls}$ & & - Expression... Clear Apply \\
\hline \multirow[t]{3}{*}{ No. } & Time & Source & Destination & Protocol Length Info \\
\hline & 6999.738000 & 172.16 .4 .10 & 172.16 .1 .10 & 160 UPDATE Message \\
\hline & 90113.856000 & 172.16 .4 .10 & 172.16 .1 .10 & 160 UPDATE Message \\
\hline
\end{tabular}

Fig. 6. Análisis conexión malla completa función extracción de etiquetas MPLS

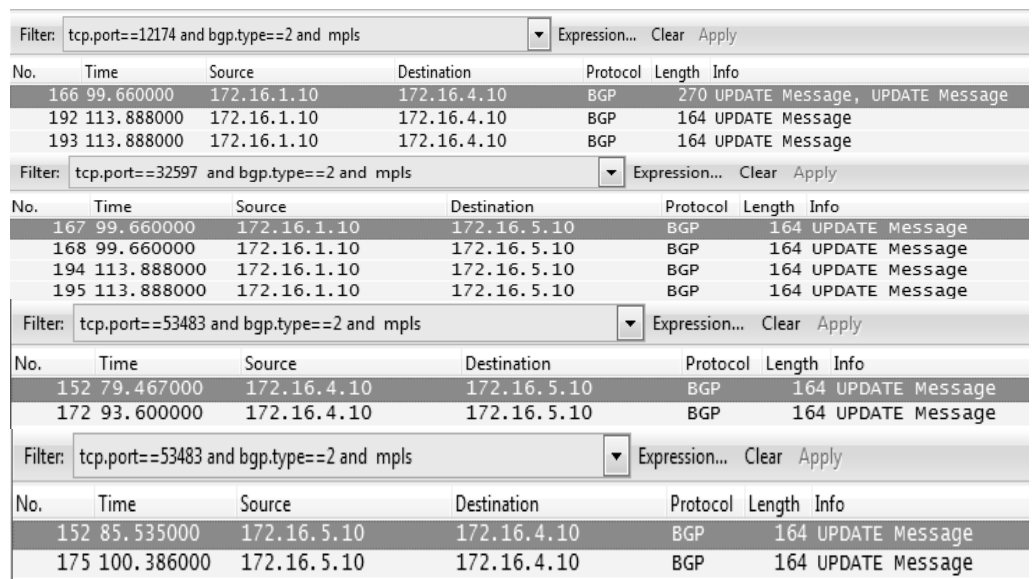

Fig. 7. Análisis conexión malla completa función para agregar etiquetas MPLS

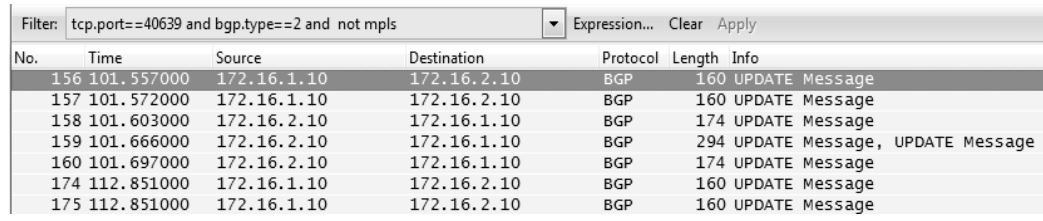

Fig. 8. Análisis conexión rutas reflejadas función extracción de etiquetas MPLS

$\sum_{i \in C} d(i, r(i))=641$ Bytes 


$$
\sum_{i \in R} \sum_{j \in R} \frac{d(i, j)}{2}=0 \text { Bytes }
$$

Como se observa en la Fig. 9, se desarrolló un análisis con filtros en wireshark, validando las conexiones BGP UPDATE que tienen encapsulamiento MPLS desarrollado (7) y (8).

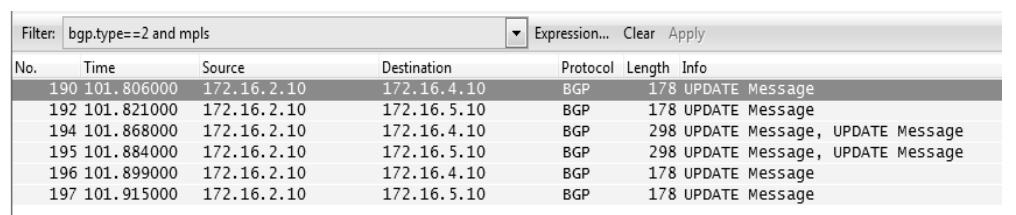

Fig. 9. Análisis conexión rutas reflejadas función para agregar de etiquetas MPLS

$$
\begin{aligned}
& \sum_{i \in C} d(i, r(i))=654 \text { Bytes } \\
& \sum_{i \in R} \sum_{j \in R} \frac{d(i, j)}{2}=0 \text { Bytes }
\end{aligned}
$$

\section{CONCLUSIONES}

Con el escenario propuesto, se logra evaluar los costos del algoritmo de enrutamiento BGP a nivel de los routers de borde de la red MPLS/BGP/VPN en el establecimiento de VRFs; para las configuraciones malla de completa y router reflector. Evaluando al wireshark como excelente herramienta en el análisis de los encapculamiento de MPLS, y en la validación del modelo matemático. Se obtuvo un $31 \%$ de mejora a nivel de anuncios en los costos del algoritmo, en la configuración de rutas reflejadas en la función de encapsulamiento MPLS. La función extracción de rutas tiene una ventaja importante para los routers de borde ya que en la configuración de rutas reflejadas esta función es realizada por el router reflector realizando una mejora en el rendimiento y en asociación de VRFs de los clientes de la red. 


\section{REFERENCIAS}

Bates T., C. E. (2006). BGP Route Reflection: An Alternative to Full Mesh Iternal BGP (IBGP)-RFC 4456. RFC.

Eason G, N. B. (1955). On certain integrals of Lipschitz-Hankel type involving products of Bessel functions. London: Phil. Trans. Roy. Soc.

Feamster N., B. H. (2005). Some Foundational Problems in Interdomain Routing. ACM SIGCOMM Workshop on hot topics in Networks.

FengJie, S. Q. (2011). Real-time signal time delay analysis of WAMS based on MPLS VPN technology. Advanced Power System Automation and Protection (APAP).

Gottlied J, G. A. (2003). Automated Provisioning of BGP Customers. AT\&T.

Li, Y. R. (1995). A Border Gateway Protocol 4 (BGP-4)-RFC 1771. RFC .

Liwen He, B. P. (2008). Pure MPLS Technology Availability, Reliability and Security. Third International Conference on ARES 08.

Marques, P. (2005). BGP Route Reflection in Layer 3 VPN Networks. Juniper networks.

Minei I, M. P. (2007). Scalability Considerations in BGP/MPLS IP VPNs Communications Magazine. IEEE.

Ming S, W. W. (2012). Engineering analysis and research of MPLS VPN. Strategic Technology (IFOST).

Palmiery, F. (2003). VPN scalability over high performance backbones Evaluating MPLS VPN against traditional approaches. Proceedings of the Eighth IEEE International Symposium on Computers and Communication (ISCC'03).

Peng, J. (2009). VPLS Technology Research and Application in the Architectures of E-Government Network. Management of e-Commerce and e-Government.

Pico, J. F. (2008). MPLS-VRF integration: forwarding capabilities of BGP/MPLS IP VPN in GNU/Linux. ONDM International Conference on.

Rekhter, E. R. (2006). BGP/MPLS IP Virtual Private Networks (VPNs)RFC 4364. RFC.

Wei, Y., Fang, Z., Dai, Z., Han, X., \& Xu, F. (2006). A MPLS and VPN Based eGovernment System. Computing CIC .

Yuri Breitbart Minos Garofalakis, A. G. (2003). Optimizing IBGP Route Reflection Network. University of Illinois at Urbana-Champaign. 